\title{
The relative importance of competition and predation in environment characterized by resource pulses - an experimental test with a microbial community
}

Teppo Hiltunen ${ }^{1 *}$ and Jouni Laakso ${ }^{2}$

\begin{abstract}
Background: Resource availability and predation are believed to affect community dynamics and composition. Although the effects of resource availability and predation on prey communities are usually studied in isolation, these factors can also have interactive effects, especially since the outcome of competition under shared predation is expected to depend on resource availability. However, there are few experimental studies that test the interactive roles of resources and predation on dynamics of more complex multispecies communities. Here, we examine the importance of competition and predation on microbial community dynamics in a resource pulse environment.

Results: We manipulated resource availability and predation simultaneously in a microbial microcosm experiment, where a bacterial community was exposed to the protozoan predator Tetrahymena thermophila in three different resource concentrations (low, intermediate and high). The prey community consisted of three heterotrophic bacterial species: Bacillus cereus, Serratia marcescens and Novosphingobium capsulatum, all feeding on a shared plant detritus medium. In fresh culture media, all species grew in all resource concentrations used. However, during experiments without any addition of extra resources, the existing resources were soon depleted to very low levels, slowing growth of the three bacterial species. Prior to the microcosm experiment, we measured the competitive ability and grazing resistance, i.e. reduced vulnerability to predation, of each prey species. The three species differed in allocation patterns: in general, N. capsulatum had the best competitive abilities and B. cereus had good grazing resistance abilities. In the long-term microcosm experiment, N. capsulatum dominated the community without predation and, with predation, $B$. cereus was the dominant species in the intermediate and high resource environments.

Conclusions: Short-term, single-species assays revealed significant differences in the allocation of competitive and defensive traits among the prey species. Based on these differences, we were, to some extent, able to predict how the long-term community structure, e.g. species dominance, is modified by the resource availability and predation interaction in pulsed resource environments. Our results are consistent with theoretical predictions and also highlight the importance of interactive effects of resource competition and predation, suggesting that these factors should not be studied in isolation.
\end{abstract}

Keywords: Community dynamics, Diversity, Predator-prey interaction, Resource competition, Resource pulse, Tetrahymena thermophila, Trophic dynamics

\footnotetext{
* Correspondence: teppo.hiltunen@helsinki.fi

'Department of Food and Environmental Sciences / Microbiology and Biotechnology, University of Helsinki, P.O. Box 65, 00014 Helsinki, Finland Full list of author information is available at the end of the article
} 


\section{Background}

The resource competition theory predicts that competition will reduce species abundance and ultimately lead to dominance by a single species in cases where there is a constant supply of a single limiting resource [1]. However, resource availability is seldom constant in natural environments; instead, the frequency and magnitude of resource supply often varies resulting in the alternation of low and high resource availability [2,3]. Perhaps the most drastic changes in resource availability are caused by resource pulses. Resource pulses are defined as low frequency temporal events leading to increased resource availability with a short duration and a large magnitude [4]. The temporal variability in resource supply during resource pulse events makes predicting the outcome of resource competition and community dynamics more challenging. One way to approach this problem is to investigate the characteristics related to resource use among the individual species in the community. In general, a species' ability to grow can differ between low and high resource environments so that the competitive dominance between species changes as a function of the resource concentration. In environments where resource pulses occur, rapid increases in resource concentrations can favor high resource specialists since their densities can respond rapidly to increasing resources. In contrast, long periods of low resource concentration can favor species that are able to maintain positive growth rates with very limited resources. Therefore, temporal changes in resource availability may allow different species to co-exist in the communities $[1,5]$.

Predation, in addition to resource availability, is a major force shaping community structure. Predation often greatly reduces prey population density and alters community composition and species diversity [6,7]. Predation can have a positive effect on prey community diversity when predators feed more on superior competitors, which would, without predators, dominate the community [8-10]. Furthermore, many studies have shown that grazing resistance is often costly and the cost is seen as reduced competitive ability $[11,12]$. The effect of resources and predation on prey community is often linked. The effect of predation on prey community composition depends on resource availability [13-16]. Using a theoretical approach, Holt et al. (1994) found that traits related to resource competition are more important for prey fitness when resources are scarce. However, when resources are more abundant, traits related to grazing resistance can play a more significant role in prey fitness [16].

The aim of this study was to investigate the relative roles of resource competition and predation on prey community dynamics after a resource pulse. In an earlier study [17] with the same system, fluctuating resource supply and predation allowed different prey species to prevail under different fluctuation regimes through trade-offs between competitive ability and grazing resistance. The present study continued this work by investigating the community dynamics in an environment where resources do not fluctuate but are provided as a single large-scale pulse and are thereafter depleted by the bacterial community. We tested how the amount of resources (intensity of the resource competition) and predation by the protozoan predator $T$. thermophila affect the population dynamics and composition of an aquatic bacterial prey community. To answer these questions, we first conducted a short-term growth and feeding experiment to estimate the competitive ability and grazing resistance of each prey species. All measurements were conducted in three resource concentrations, low, intermediate and high. We used short-term population growth rate as an estimate of competitive ability and grazing resistance as an estimate of biomass reduction by predators. To investigate long-term community dynamics, we then conducted a 21-day long microcosm experiment where a three-species prey community was cultured in three resource concentrations with and without predators. During the experiment, we monitored population densities and prey community composition with direct microscope counts and a dilution plating method. The experimental set up simulated a resource pulse and we monitored the resultant community dynamics. We predicted that resources and predation should have a substantial effect on the prey community in the microcosm experiment, based on theory $[6,7]$ and previous studies of similar microbial systems [9,10,17-19]. We also predicted that traits of individual species should explain community dynamics with traits related to competition being more important in the absence of predators and traits related to grazing resistance being more important when predators are present. In our case, we concluded, based on competitive ability and grazing resistance measurements, that $N$. capsulatum was the best resource competitor in low and intermediate resource concentrations and B. cereus had overall good grazing resistance abilities. The role of predation is considered to be less important in low resource environments where resource competition is more important [16]. One reason behind this prediction is that in less productive environments predator population sizes are also smaller and thus predation pressure is lower. The microcosm community experiment confirmed our predictions and the prey species that was a better resource competitor was dominant in the absence of the predator and the more grazing resistant species dominated in the presence of predation. Furthermore, the role of predation was less significant in the low resource environment where traits related to competitive ability seem to be more important in determining the identity of the dominant species.

\section{Results}

For the simplified results summary see Table 1. 
Table 1 Summary of the results from the trait and community experiments

\begin{tabular}{|c|c|c|c|c|}
\hline \multirow[b]{2}{*}{ Resource level } & \multicolumn{2}{|c|}{ Predator absent } & \multicolumn{2}{|c|}{ Predator present } \\
\hline & Best competitive ability & $\begin{array}{l}\text { Dominant species in } \\
\text { the community experiment }\end{array}$ & Best grazing resistance & $\begin{array}{l}\text { Dominant species in } \\
\text { the community experiment }\end{array}$ \\
\hline Low & N.C & N.C & B. $c / S . m$ & N.C \\
\hline Intermediate & N. C & N. C & B. C / N.C & B. $C$ \\
\hline High & S. $m$ & N. C & B. C /N.C & B. C \\
\hline
\end{tabular}

Species codes: B. cereus (B. C), S. marcescens (S. $m$ ) and N. capsulatum (N. c).

\section{Trait measurements}

\section{Competitive ability}

Species growth rates increased with increasing resource concentration, but the competitive rank between species varied among resource concentrations (Figure $1 \mathrm{~A}$; resource treatment: $\mathrm{F}_{2.98}=85.7 ; \mathrm{p}<0.0001$, species identity $\times$ resource treatment: $\left.\mathrm{F}_{6.98}=36.7 ; \mathrm{p}<0.0001\right)$. We found that $N$. capsulatum had a higher growth rate than the two other species in low and intermediate resource concentrations when comparing species growth rates in different resource concentrations (Figure 1A; homogenous subsets; low resource concentration: $\mathrm{a}: \mathrm{p}=0.10$; $\mathrm{b}$ : $\mathrm{p}=$

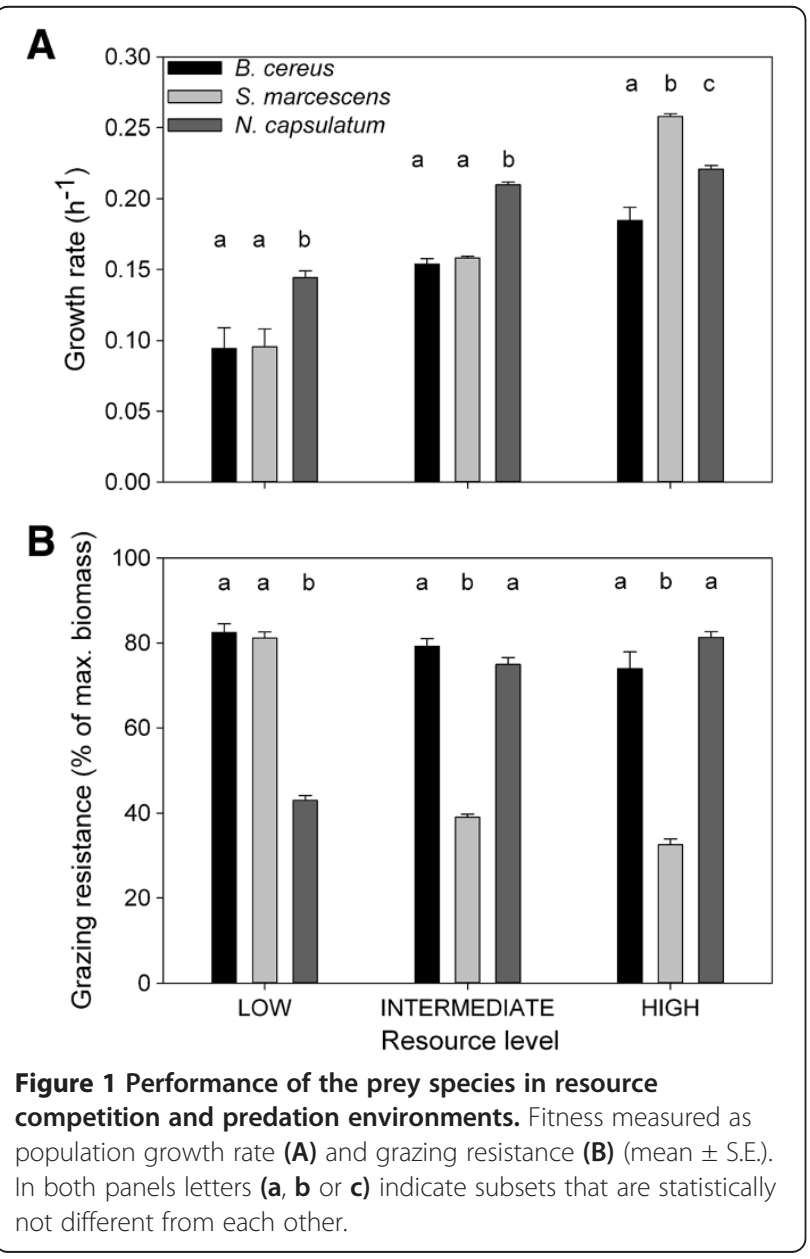

1.00; intermediate resource concentration: $\mathrm{a}: \mathrm{p}=0.43$; $\mathrm{b}$ : $\mathrm{p}=1.00)$. S. marcescens had the highest growth rate, $N$. capsulatum had the second highest and B. cereus had the lowest, in high resource concentrations (Figure 1A; all species in different homogenous subsets).

\section{Grazing resistance}

Grazing resistance, as with population growth rates, was different among species and rank among species depended on the resource concentration (Figure 1B; species identity: $\mathrm{F}_{3.98}=79.4 ; \mathrm{p}<0.0001$, resource treatment: $\mathrm{F}_{2.98}=19.5$; $\mathrm{p}<0.0001)$. The grazing resistance of $S$. marcescens decreased with increasing resource concentration, the grazing resistance of $N$. capsulatum increased, and resource concentration had no effect on the grazing resistance of B. cereus (Figure $1 \mathrm{~B}$, species identity $\times$ resource treatment: $\left.\mathrm{F}_{6.98}=75.2 ; \mathrm{p}<0.0001\right)$. We found that $N$. capsulatum had a lower grazing resistance in low resource concentrations than the two other species (Figure 1B; homogenous subsets; $\mathrm{a}: \mathrm{p}=0.84 ; \mathrm{b}: \mathrm{p}=1.00)$. In intermediate and high resource concentrations, $S$. marcescens had lower grazing resistance than the other species (Figure 1B, homogenous subsets; intermediate resource concentration; $\mathrm{a}: \mathrm{p}=0.13$; $\mathrm{b}: \mathrm{p}=1.00$; high resource concentration; $\mathrm{a}: \mathrm{p}=0.12 ; \mathrm{b}: \mathrm{p}=1.00$ ).

\section{Community experiment \\ Population densities}

The total number of prey individuals was lower when predators were present in all resource concentrations and each concentration produced low, intermediate and high population densities (Figure 2; predator treatment: $\mathrm{F}_{1.18}=$ 413; $\mathrm{p}<0.0001$, resource treatment: $\mathrm{F}_{2.18}=440 ; \mathrm{p}<0.0001$, all in different homogenous subsets). Predator population density was lower in low resource concentrations compared to intermediate and high resource concentrations (Figure 2; resource treatment: $\mathrm{F}_{2.9}=30.1 ; \mathrm{p}<0.0001$, intermediate and high resource concentrations in the same homogenous subset, $\mathrm{p}=0.11$ ). Furthermore, predator population density declined over time in all resource concentrations (time: $\mathrm{F}_{1.9}=256 ; \mathrm{p}<0.0001$ ).

\section{Species proportions in the prey community}

The effects of predation on prey community composition differed along the resource concentration axis. The 


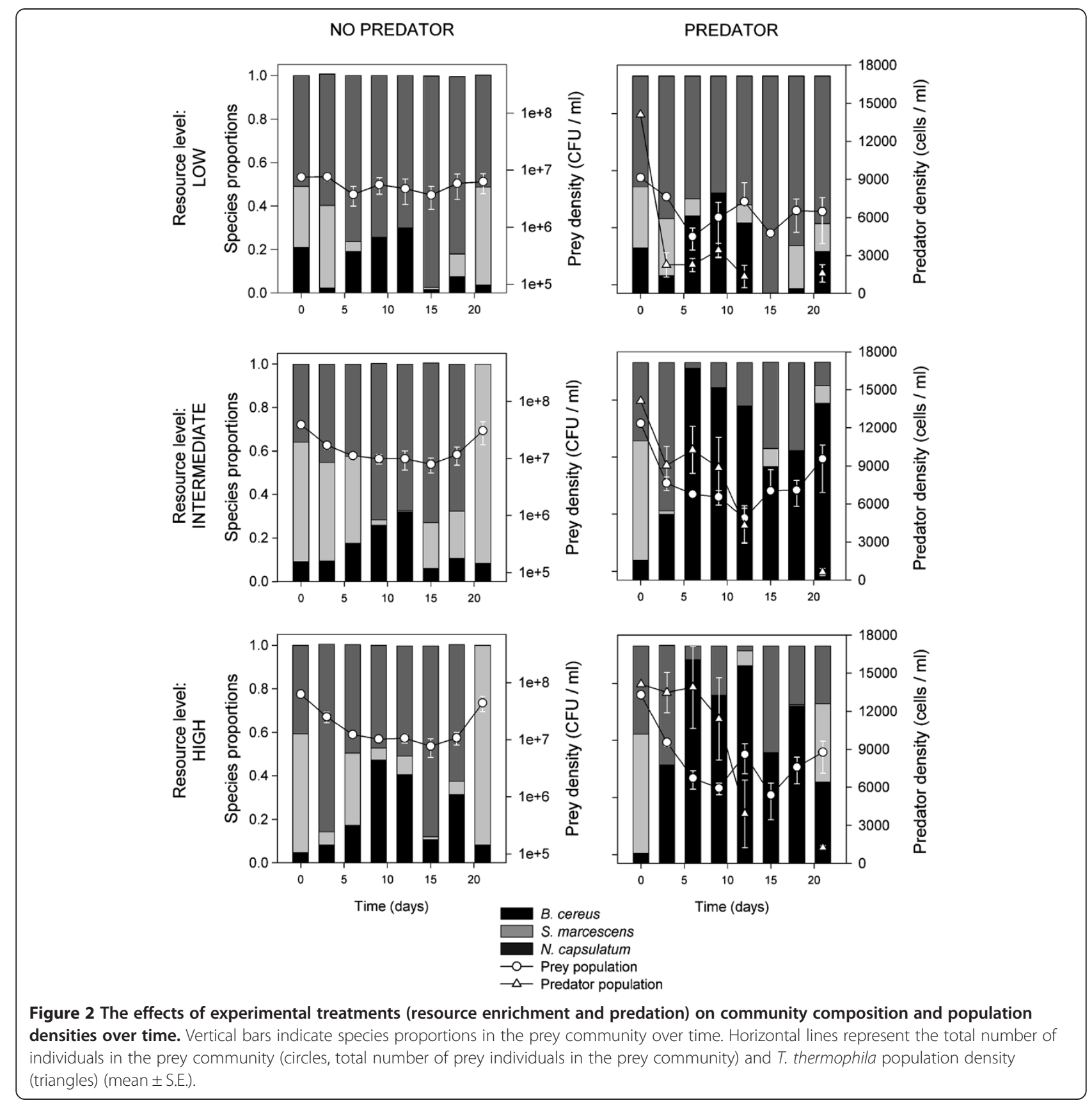

proportion of $N$. capsulatum was higher than the two other species in the low resource concentrations, both with and without predators (Figure 2; predators absent: $\mathrm{F}_{2.9}=58.8 ; \mathrm{p}<0.0001$, S.m and B.c in the same homogenous subset, $\mathrm{p}=0.93$; predators present: $\mathrm{F}_{2.9}=35.5$; $\mathrm{p}<0.0001$, S. $m$ and B.c in the same homogenous subset, $\mathrm{p}=0.55$ ). In intermediate resource concentrations, the proportions of all species were different from each other; the proportion of $N$. capsulatum was the highest in treatments without predators, $B$. cereus was the lowest, and the proportion of $B$. cereus in treatments with predators was the highest and S. marcescens the lowest (Figure 2; predators absent: $\mathrm{F}_{2.9}=25.0 ; \mathrm{p}<0.0001$, predators present: $\mathrm{F}_{2.9}=63.8 ; \mathrm{p}<0.0001$, in both, all species in different homogenous subsets). The proportion of $N$. capsulatum was higher than the other two species in high resource concentrations without predators (Figure 2; $\mathrm{F}_{2.9}=15.2 ; \mathrm{p}=$ 0.001 , S. $m$ and B.c in the same homogenous subset, $\mathrm{p}=$ $0.65)$. The proportion of $B$. cereus was the highest and $S$. marcescens was the lowest in high resource concentrations with predators (Figure 2; $\mathrm{F}_{2.9}=116 ; \mathrm{p}<0.0001$, all species in different homogenous subsets). 


\section{Discussion}

Competition for shared resources and predation are usually among the most important factors driving prey community dynamics [20]. To investigate the effects of these two factors on community dynamics after a resource pulse, we conducted a microbial microcosm experiment where we manipulated the intensity of resource competition with resource pulse concentration as well as the presence of predation. In order to predict and understand the outcome of community dynamics, we measured growth and defense related traits of each prey species prior to the long-term experiment. Based on these measurements, we were able to categorize $N$. capsulatum as a good resource competitor, $B$. cereus being more resistant against grazing and $S$. marcescens as an opportunist specialized in high resource environments (Figure 1). Results from the community experiment support this view: without predators in all resource concentrations and also with predators in low resource concentration, $N$. capsulatum was the dominant species (Figure 2). However, predation hindered the dominance of $N$. capsulatum; in intermediate and high resource concentrations with predation, the inferior competitor but more grazing resistant $B$. cereus was the dominant species (Figure 2). See Table 1 for a summary of the results.

Temporal changes in resource availability, such as resource pulses, are known to promote species coexistence [4]. This enhanced diversity is often explained by interspecific differences in the ability to maintain positive growth under low resource conditions and rapid growth when resources are abundant [21-24]. The role of fluctuating resources on species co-existence and community dynamics was tested in a previous study [17] using the same microbial community as here. The main finding in that study was that S. marcescens was the dominant prey species across all treatments [17], a qualitatively different result compared to the present study. However, in the Hiltunen et al. 2012 study, the inflow of fresh media, having the same concentration as "high" in the current study, was a frequent event (on average $\sim 80 \%$ of the media was replaced daily), enabling S. marcescens to dominant the community. A likely reason why $S$. marcescens was able to dominate in the previous study was its high growth rate in the high resource concentration of the fresh culture media (Figure 1A: high resource concentration). In the current study, S. marcescens was not the dominant species in any of the treatments. $S$. marcescens seem to be a high resource specialist and in our current experiment, even in the initially high resource concentration, the availability of resources declined rapidly since there was no further input of new resources and the consumers depleted the existing resources. Thus, high resource specialists will experience a fitness benefit only for a short period of time and after a while species specialized in the use of low resource concentrations, such as $N$. capsulatum in our experiment, will dominate the communities. However, we found that in the intermediate and high resource concentrations without predators $S$. marcescens became the dominant species by the last time point and also increased its proportion substantially in the low resource environment during the last three time points. This is a result that cannot be explained with the competitive ability measurements. One explanation for this change in dominance after 21 days is the change in the resource quality. Pekkonen et al. (2012) studied resource mediated indirect facilitative and inhibitory interactions between $S$. marcescens and $N$. capsulatum, competing for the same plant detritus resource as used in our experiment. They found, among other things, that $S$. marcescens benefited from the presence of consumed growth media in contrast to $N$. capsulatum. To investigate similar questions, Lawrence et al. (2012) [25] used a nuclear magnetic resonance (NMR) spectroscopy to study the qualitative changes in a complex, organic plant based culture media. This approach could also have been useful in our study for investigating qualitative changes in our culture media and explain why S. marcescens was able to increase its proportion at the end of the experiment. However, based on data that we have now, we can conclude that in our resource pulse environment, temporal changes in the resource availability might not only have been quantitative but also qualitative. Even though we do not have direct evidence, this type of complex facilitative interaction could explain why the inferior competitor in low concentrations of fresh growth media could dominate at the end of the experiment when concentrations of the original resources were bound to be extremely low. However, this also makes predicting the competitive outcome based solely on growth rate measurements more challenging.

Predation is generally predicted to have a positive effect on coexistence among prey species when predators prevent the exclusion of more resistant but less competitive prey types $[6,7,16,26]$. The community data presented here is a good example of this scenario. Without predators, $N$. capsulatum was the dominant species; however, with predators in intermediate and high resource concentrations, the good resource competitor $N$. capsulatum was no longer able to dominate the prey community. B. cereus, an inferior resource competitor but an inherently more grazing resistant species, dominated the prey community in these conditions.

Interestingly, we found an interactive effect between resource concentration and predation treatments so that in low resource environments $N$. capsulatum was the dominant prey species instead of the more grazing resistant $B$. cereus. Holt et al. (1994) were the first to use a theoretical approach to investigate the interplay of exploitative and apparent competition and described the shift from dominance of exploitative to apparent competition along the enrichment gradient. Our experimental findings described above 
are consistent with the predictions by Holt et al. We observed, as the theory predicts, a shift in dominance from the superior resource competitor ( $N$. capsulatum) to the grazing-resistant prey (B. cereus) along an enrichment gradient when the prey community was exposed to predation. Changes in the relative importance of competition and predation related traits along an enrichment axis might explain this finding in our experiments. When resources are extremely limited, traits related to competitive ability are disproportionally important and the importance of these traits may override any benefits that the higher grazing resistance can provide. Also, the fact that predator population density and, therefore, predation pressure was lower in the low resource environment could have contributed to making competitive ability a more important factor in determining the dominant prey species.

\section{Conclusions}

We found that in our experimental bacterial communities, either resource availability or ciliate predation determined the dominant species. This is in line with theoretical predictions $[1,2,6,7,21,23,26,27]$. In most cases, we were able to predict the identity of the dominant species based on trait characteristics measured prior to the community experiment in single species short-term assays. We also found that resource concentration and predation treatments strongly interacted so that in low resource environments competitive ability was the main factor determining the community composition.

\section{Methods}

\section{Study species and culture media}

Our experimental prey community consisted of three heterotrophic bacterial species: Bacillus cereus (ATCC 14579), Serratia marcescens (ATCC 13880) and Novosphingobium capsulatum (ATCC 14666), all feeding on shared plant detritus medium. All strains were obtained from the American Type Culture Collection [28]. The criteria for selecting these three bacterial species included positive growth on both the nutrient broth agar and liquid prey culture medium and that the species were distinguishable based on their colony color and morphology. The source of organic carbon in our liquid prey culture medium was filtered plant detritus (hay extract, Ward's natural science, Rochester, NY), for a detailed recipe and methods for culture media preparation see $[17,18]$. Three final concentrations of plant detritus used throughout experiment were $0.215,1.075$ and $2.15 \mathrm{mg} / \mathrm{l}$ and they are referred hereafter as low, intermediate and high resource concentrations. When the experiments were initiated, the bacterial strains (stored in $50 \%$ glycerol at $-70^{\circ} \mathrm{C}$ ) were first thawed and then cultivated on nutrient broth agar (10 g nutrient broth, $2.5 \mathrm{~g}$ yeast extract and $15 \mathrm{~g}$ agar (all Sigma-Aldrich) in 1 liter of $\mathrm{dH}_{2} \mathrm{O}$.
As a predator, we used a ciliated protozoa, Tetrahymena thermophila (ATCC 30008), which is an asexual strain consisting of only a single mating type and has been widely used in experimental microbial ecology $[8,9,17,29-31] T$. thermophila was cultured in a proteose peptone-yeast medium (10 g nutrient broth, 2.5 g yeast extract (SigmaAldrich) in 1 liter of $\mathrm{dH}_{2} \mathrm{O}$ ) in controlled laboratory conditions prior to the experiment. All experiments were carried out at $25 \pm 1^{\circ} \mathrm{C}$.

\section{Estimating competitive ability and grazing resistance}

We conducted a short-term growth and feeding experiment to estimate the competitive ability and grazing resistance of each prey species. As an estimate of competitive ability, we used population growth rate and grazing resistance estimated as biomass reduction by predators. Measurements were conducted in each of the three resource concentrations (low, intermediate and high).

When measuring the population growth rates, $350 \mu \mathrm{l}$ of fresh culture medium was inoculated with a small amount of bacteria ( $10 \mu \mathrm{l}$ containing $\sim 10^{\wedge} 6$ individuals). Then, the population was allowed to grow for 96 hours and the initial period when resources were not limiting was used to estimate the maximum growth rate (see below). When measuring grazing resistance, we used the same bacterial cultures that were allowed to reach carrying capacity ( $96 \mathrm{~h}$ ) before adding $10 \mu \mathrm{l}$ of centrifuged and washed predator stock in $\mathrm{pH} 7.5$ phosphate buffer containing $~ 100$ predator individuals. We monitored bacterial biomass (optical density) for another 96 hours which allowed predators to reach a high density and prey population was simultaneously grazed down. This reduction of prey biomass (percent grazed biomass from initial biomass prior to the addition of the predators) was then used as an estimate of the grazing resistance. All treatments were replicated nine times.

Population growth and predator-induced decline was measured with Bioscreen $C$ spectrophotometer (Growth Curves AB Ltd, Finland) where optical density of each well was measured at $480-580 \mathrm{~nm}$ wavelengths at five minute intervals. Population growth rate was calculated as the slope of the linear regression of natural logarithms of population biomass versus time when the population grew at its maximal rate. This methodology in bacterial trait measurements has been used successfully in previous studies with similar systems (see e.g. $[9,17,18]$ ).

\section{Microcosm community experiment}

To study community dynamics after a resource pulse, we conducted a factorial microcosm experiment in batch cultures where bacterial communities were cultured in three resource levels (low, intermediate and high), with and without predators. Each treatment was replicated four times.

The microcosms were contained in $250 \mathrm{ml}$ polycarbonate Erlenmeyer flasks (Corning) and experimental 
communities were assembled as follows: each bacterial species was grown separately for 96 hours in liquid medium and then mixed in even proportions $10 \mathrm{ml}$ each and $750 \mu \mathrm{l}$ of centrifuged predator containing $\sim 7.5 \times 10^{\wedge} 4$ individuals was added to the predation treatments. At the beginning of the experiment, the total volume of liquid in each microcosm was $30.75 \mathrm{ml}$. The experiment was carried out for 21 days and sampling was conducted every three days by removing a $1 \mathrm{ml}$ sample from each microcosm under sterile conditions.

A Lugol's solution was used to fix $200 \mu \mathrm{l}$ of the $1 \mathrm{ml}$ sample and the predator population densities were determined automatically from the digitized images using an image recognition script (for detailed methods see [29]). Two predator samples, days 15 and 18, were misplaced and treated as missing data. The prey species population sizes were determined with serial dilution plating and by counting the colony forming units (CFU's) cultured on petri dishes containing nutrient broth agar.

\section{Statistical analysis}

The effect of resource concentration and predation on population growth rates and grazing resistance were analyzed with a two-way ANOVA. Post-hoc Tukey comparisons were performed to test pairwise interactions. In the community experiment, the effect of treatments on population densities and species proportions were analyzed with repeated measurements ANOVA (RMANOVA). The requirements of the RMANOVA only allowed the analysis of the first five samples of the predator population data due to the loss of samples from days 15 and 18. Population growth rates $\left(h^{-1}\right)$ were calculated as $\mathrm{r}=\ln \left(\mathrm{N}_{\mathrm{t}+1} / \mathrm{N}_{\mathrm{t}}\right) / \mathrm{t}$ where $\mathrm{N}_{\mathrm{t}}$ is population size at time, $t$. All analyses were performed with PASW statistics (SPSS Inc. Chicago IL, v. 20.0) software.

\section{Abbreviations}

CFU: Colony forming unit; ANOVA: Analysis of variance; RMANOVA: Repeated measures analysis of variance; B. c.: Bacillus cereus; S.m.: Serratia marcescens; N. c.: Novosphingobium capsulatum.

\section{Authors' contributions}

$\mathrm{TH}$ and $\mathrm{J}$ designed the study. TH conducted the experiments, designed and performed the statistical analyses and drafted the manuscript. $J L$ supervised the research and revised the manuscript. Both authors read and approved the final version of the manuscript.

\section{Acknowledgements}

We thank M. Niskanen for assistance in the lab and K. Viipale for the insightful comments during the experiment procedure. We also thank D. Fewer and $\mathrm{H}$. Devlin for editing the language. The study was funded by the Academy of Finland (project \#106993) and the Center of Excellence in Evolutionary Biology.

\section{Author details}

1 Department of Food and Environmental Sciences / Microbiology and Biotechnology, University of Helsinki, P.O. Box 65, 00014 Helsinki, Finland. 2Department of Biosciences / Integrative Ecology Unit, University of Helsinki, P.O. Box 65, 00014 Helsinki, Finland.
Received: 6 November 2012 Accepted: 29 August 2013

Published: 3 September 2013

\section{References}

1. Hardin G: The competitive exclusion principle. Science 1960, 131:1292-1297.

2. Rosenzweig ML: Species Diversity in Space and Time. Cambridge: Cambridge University Press; 1995.

3. Ostfeld RS, Keesing F: Pulsed resources and community dynamics of consumers in terrestrial ecosystems. Trends Ecol Evol 2000, 15:232-236.

4. Yang $L H$, Bastow JL, Spence KO, Wright AN: What can we learn from resource pulses. Ecology 2008, 89:621-634.

5. Tilman D: Resource competition and community structure. Princeton, New Jersey: Princeton University Press; 1982.

6. Chesson P: Mechanisms of maintenance of species diversity. Annu rev ecol syst 2000, 31:343-366.

7. Chase JM, Abrams PA, Grover JP, Diehl S, Chesson P, Holt RD, Richards SA Nisbet RM, Case TJ: The interaction between predation and competition: a review and synthesis. Ecol Lett 2002, 5:302-315.

8. Meyer JR, Kassen R: The effects of competition and predation on diversification in a model adaptive radiation. Nature 2007, 446:432-435.

9. Friman $V$ - $P$, Hiltunen $T$, Laakso J, Kaitala V: Prey resource availability drives evolution of predator prey interaction. Proc R SOC B 2008, 275:1625-1633.

10. Friman V-P, Laakso J: Pulsed resource dynamics constrain the evolution of predator-prey interactions. Am Nat 2011, 177:334-345.

11. Yoshida T, Jones LE, Ellner SP, Fussmann GF, Hairston NG Jr: Rapid evolution drives ecological dynamics in a predator-prey system. Nature 2003, 424:303-306.

12. Yoshida T, Hairston NG Jr, Ellner SP: Evolutionary trade-off between defence against grazing and competitive ability in a simple unicellular alga, Chlorella vulgaris. Proc R SOC B 2004, 271:1947-1953.

13. Jiang $L$, Krumins JA: Emergent multiple predator effects in an experimental microbial community. Ecol Res 2006, 21:723-731.

14. Worm B, Lotze HK, Hillebrand H, Sommer U: Consumer versus resource control of species diversity and ecosystem functioning. Nature 2002, 417:848-851.

15. Hall RA, Meyer JR, Kassen R: Selection for predator resistance varies with resource supply in a model adaptive radiation. Evol Ecol Res 2008, 10:735-746.

16. Holt RD, Grover J, Tilman D: Simple rules for interspecific dominance in systems with exploitative and apparent competition. Am Nat 1994, 144:741-771.

17. Hiltunen T, Friman V-P, Kaitala V, Mappes J, Laakso J: Predation and resource fluctuations drive eco-evolutionary dynamics of a bacterial community. Acta Oecol 2012, 38:77-83.

18. Hiltunen $T$, Laakso J, Kaitala V, Suomalainen L-R, Pekkonen M: Temporal variability in resource availability affects diversity of aquatic bacterial communities. Acta Oecol 2008, 33:291-299.

19. Pekkonen $\mathrm{M}$, Korhonen J, Laakso J: Increased survival during famine improves fitness of bacteria in pulsed resource environment. Evol Ecol Res 2011, 13:1-18.

20. Begon M, Townsend CR, Harper JL: Ecology: From Individuals to Ecosystems. Oxford: Wiley-Blackwell; 2005

21. Grover JP: Resource competition. New York: Chapman \& Hall; 1997.

22. Kirk K: Competition in variable environments: experiments with planktonic rotifers. Freshwater biol 2002, 47:1088-1096.

23. Sommer U, Worm B: Competition and coexistence, Volume 161. Berlin: Springer; 2002.

24. Angert $A L$, Huxman $T E$, Chesson $P$, Venable DL: Functional tradeoffs determine species coexistence via the storage effect. PNAS 2009, 106:11641-11645.

25. Lawrence D, Fiegna F, Behrends V, Bundy JG, Phillimore AB, Bell T, Barraclough TG: Species interactions alter evolutionary responses to a novel environment. Plos Biol 2012, 10:e1001330.

26. Gendron RP: Models and mechanism of frequently-dependent predation. Am Nat 1987, 130:603-623.

27. Leibold MA: A graphical model of keystone predators in food webs: trophic regulation of abundance, incidence, and diversitypatterns in communities. Am Nat 1996, 147:784-812.

28. LGC Promochem, protistology collection (search for strain numbers). http:// www.lgcstandards-atcc.org 
29. Laakso J, Löytynoja K, Kaitala V: Environmental noise and population dynamics of the ciliated protozoa Tetrahymena thermophila in aquatic microcosms. Oikos 2003, 102:663-671.

30. Ketola T, Laakso J, Kaitala V, Airaksinen S: Evolution of Hsp90 expression in Tetrahymena thermophila (protozoa, ciliata) populations exposed to thermally variable environments. Evolution 2004, 58:741-748.

31. Friman V-P, Buckling A: Effects of predation on real-time host-parasite coevolutionary dynamics. Ecol Lett 2013, 16:39-46.

doi:10.1186/1472-6785-13-29

Cite this article as: Hiltunen and Laakso: The relative importance of competition and predation in environment characterized by resource pulses - an experimental test with a microbial community. BMC Ecology 2013 13:29

\section{Submit your next manuscript to BioMed Central and take full advantage of:}

- Convenient online submission

- Thorough peer review

- No space constraints or color figure charges

- Immediate publication on acceptance

- Inclusion in PubMed, CAS, Scopus and Google Scholar

- Research which is freely available for redistribution 